\title{
Comportamentos internalizantes: associações com habilidades sociais, práticas educativas, recursos do ambiente familiar e depressão materna
}

\author{
Alessandra Turini Bolsoni-Silva \\ Universidade Estadual Paulista, SP, Brasil \\ Sonia Regina Loureiro \\ Edna Maria Marturano \\ Faculdade de Medicina de Ribeirão Preto, SP, Brasil
}

\section{Resumo}

Problemas de comportamento internalizantes são subidentificados e carecem de estudos que investiguem diversas variáveis simultaneamente. Os objetivos são comparar e correlacionar indicadores de práticas educativas, recursos do ambiente familiar, repertório comportamental infantil e depressão materna de crianças com e sem problemas internalizantes, diferenciadas por sexo e escolaridade. Participaram 32 mães de crianças com problemas internalizantes e 32 sem problemas, que foram pareadas por sexo e escolaridade. Foram utilizados instrumentos validados para mensurar as variáveis do estudo, cujos dados foram comparados e correlacionados. Os resultados são: (a) práticas negativas foram mais frequentes no grupo com problemas e, habilidades sociais, no grupo sem problemas; (b) práticas negativas correlacionaram-se a problemas e práticas positivas às habilidades sociais e recursos do ambiente; (c) quanto maior a depressão materna, menores as habilidades sociais infantis; (d) crianças escolares têm mais problemas de ansiedade que pré-escolares. Discute-se sobre as implicações das variáveis estudadas para os comportamentos internalizantes.

Palavras-chaves: Depressão infantil; Ansiedade infantil; Práticas educativas; Recursos do ambiente; Habilidades sociais; Depressão.

\section{Internalizing behavior problems and associations with social skills, educative practices, familiar resources and maternal depression}

\begin{abstract}
Internalizing behaviors are under-identified and scarcely studied in association with more than one variable simultaneously. The objectives are to compare and correlate indicators of parental practices, family resources, child behavior, and maternal depression, in children with or without internalizing problems, differentiated by gender and schooling. Participants were 32 mothers of children with internalizing problems and 32 mothers of children without problems, which were matched for gender and schooling. Validated instruments were used to measure variables of interest. Data were compared and correlated. The results are: (a) negative practices were more frequent in the problems group and social skills, in the group without problems; (b) negative practices correlated to complaints about problems and positive practices correlated to child social skills and environmental resources; (c) higher maternal depression was associated with less social skills; (d) school-aged children are more anxious than pre-schoolers. Implications of these results for understanding internalizing behaviors are discussed.
\end{abstract}

Keywords: Childhood depression; Childhood anxiety; Educational practices; Environmental resources; Social skills; Depression.

\section{Comportamientos internalizantes: asociaciones con habilidades sociales, prácticas educativas, recursos del ambiente familiar y depresión materna}

\section{Resumen}

Problemas de comportamiento internalizantes son subidentificados y faltan estudios de diversas variables simultáneamente. Los objetivos son comparar y correlacionar indicadores de prácticas educativas, recursos del ambiente familiar, repertorio comportamental infantil y depresión materna de niños con y sin problemas internalizantes, diferenciadas por sexo y escolaridad. Participaron 32 madres de niños con y 32 sin problemas internalizantes, pareadas por sexo y escolaridad. Fueron utilizados instrumentos validados para mensurar las variables, cuyos datos fueron comparados y correlacionados. Los resultados son: (a) prácticas negativas fueron más frecuentes en el grupo con problemas, y habilidades sociales en el grupo sin problemas; (b) prácticas negativas se correlacionaron a problemas y prácticas positivas a las habilidades sociales y recursos del ambiente; (c) cuanto mayor la depresión materna, menor las habilidades sociales infantiles; (d) niños escolares tienen más problemas de ansiedad que pre-escolares. Se discute sobre las implicaciones de las variables estudiadas para los comportamientos internalizantes. Palabras-clave: depresión infantil; ansiedad infantil; prácticas educativas; recursos del ambiente; habilidades sociales; depresión. 
Os problemas de comportamento internalizantes se caracterizam como distúrbios pessoais, a saber, ansiedade, retraimento, depressão e sentimento de inferioridade, enquanto os problemas externalizantes envolvem características de desafio, impulsividade, agressão, hiperatividade, favorecendo os conflitos com o ambiente (Achenbach et al., 2008). Na literatura, são poucos os estudos voltados para a temática de problemas de internalização, reconhecidamente sub identificados e sub tratados, em que pese seu impacto adverso no desenvolvimento socioemocional e na saúde (Flett \& Hewitt, 2013; Ghandour, Kogan, Blumberg \& Perry, 2010). Nesse sentido, a identificação de tais problemas na infância, mesmo os subclínicos, é importante porque esses podem, com o tempo, transformar-se na forma completa do transtorno com o qual seus sintomas se relacionam, seja de ansiedade, depressão, ou ambos, em comorbidade (Dozois \& Dobson, 2004; Flett \& Hewitt, 2013).

Do ponto de vista do desenvolvimento, problemas de comportamento internalizantes tendem a aumentar entre os anos pré-escolares e os escolares (Fanti \& Henrich, 2010). Eles têm sido associados ao sexo feminino (APA, 2000; Fanti \& Henrich, 2010), porém em grupos de crianças com diagnóstico de transtorno mental não foram encontradas diferenças de sexo (Munkvold \& Lundervold, 2011; Graziano, Geffkene \& McNamara, 2011).

Dados os efeitos nocivos dos problemas de comportamento internalizantes sobre o desenvolvimento, importa esclarecer as condições de risco a eles associadas, notadamente aquelas passíveis de controle ou mudança, assim como os mecanismos de proteção que, na presença do risco, possam atenuar ou mesmo prevenir sua manifestação, de modo a subsidiar propostas de prevenção. No âmbito pessoal, características de temperamento como inibição e temperamento difícil têm sido apontadas como precursoras de problemas internalizantes (Essau, 2004). Assim, há algum indício de que problemas de comportamento precoces predizem sintomas internalizantes na meninice (Burt \& Roisman, 2010). Em contrapartida, habilidades sociais contribuem para a atenuação de tais sintomas, engendrando mecanismos de proteção (Boyd \& Waanders, 2013; Letcher, Sanson, Smart \& Toumbourou, 2012; Wichstrom, Belsky \& Berg-Nielse, 2013).

Entre os fatores de risco ambiental, sobressaem a depressão materna (APA, 2000; Fanti \& Henrich, 2010; Pizeta, Silva, Cartafina \& Loureiro, 2013) e variáveis associadas às práticas parentais (Dozois \& Westra, 2004; Essau, 2004; Luyckx et al., 2011; Pizeta et al., 2013). Em revisão sobre o impacto da depressão materna na saúde mental das crianças, Pizeta e colaboradores (2013) encontraram evidência robusta de risco para manifestações de ansiedade e depressão infantil. Tais efeitos, segundo dados da mesma revisão, seriam intensificados na presença de interações familiares pobres e negativas, com destaque para estilos parentais negativos, rejeição, indisponibilidade emocional e punição severa. Por exemplo, Trepat, Granero e Ezpeleta (2014) verificaram que a ansiedade-depressão materna mediava a punição corporal de crianças com 3-5 anos, ao passo que Xing e Wang (2013) verificaram que práticas de punição severa prediziam problemas internalizantes em crianças de ambos os sexos e que as meninas respondiam com tais sintomas também à punição branda. Efeitos transacionais, com influências recíprocas, são sugeridos em relação ao comportamento difícil, dada a constatação de que crianças que irritam mais os adultos estão em maior risco de serem alvo de práticas negativas, gerando mais ansiedade (Hudson, Flannery-Schroeder \& Kendall, 2004).

Embora com menor ênfase que aquela dada aos fatores de risco, há indícios de que, diante de condições de risco para o desenvolvimento de problemas de comportamento internalizante, o ambiente familiar pode contribuir com mecanismos de proteção, relacionados a práticas educativas parentais positivas. No estudo de Boyd e Waanders (2013), crianças de 8 a 14 anos expostas ao risco associado à depressão materna apresentaram menos sintomas de depressão quando as mães se valiam de práticas educativas positivas, baseadas no encorajamento de comportamento apropriado e envolvimento nas rotinas diárias da criança. Luyckx et al. (2011), acompanhando crianças desde os seis anos até a adolescência, com 18 anos, encontraram trajetórias consistentemente mais baixas de problemas internalizantes no grupo de crianças cujos pais e/ou mães adotavam um estilo parental que combinava monitoramento e afeto positivo elevados com pouca disciplina inconsistente.

Há alguma evidência de efeito protetor da qualidade do ambiente familiar, em termos de recursos que promoveriam o desenvolvimento emocional, social e cognitivo da criança, tais como oportunidades de interação, passeios e rotinas regulares (Marturano, 2006). Crianças em risco mostraram menos problemas internalizantes em ambientes de melhor qualidade segundo o Inventário HOME (Kelley, Whitley \& Campos, 2011), e quando havia maior oferta de materiais de aprendizagem em casa (Rijlaarsdam et al., 2013).

Analisando-se tais relatos constata-se que os problemas de comportamento internalizantes, embora sejam considerados multideterminados, carecem de 
estudos que investiguem diversas variáveis simultaneamente, como apontado por Hudson et al. (2004) ao assinalar as dificuldades de rastreamento dos fatores de risco e de proteção para problemas de comportamento internalizantes na infância. $O$ presente estudo se insere nessa lacuna, propondo-se a avaliar simultaneamente as variáveis habilidades sociais, queixas de problemas comportamentais, indicadores de práticas educativas, recursos do ambiente familiar e depressão materna, visando identificar condições de risco e proteção para os comportamentos internalizantes.

Com base nessas considerações, têm-se como objetivos: a) comparar crianças, com e sem problemas internalizantes (pré-escolares e escolares, de ambos os sexos) quanto ao repertório comportamental infantil (habilidades sociais e queixas de problemas), os indicadores de práticas educativas (positivas e negativas), dos recursos do ambiente familiar, e de depressão materna; e b) verificar, para as crianças com problemas internalizantes, as possíveis associações das variáveis habilidades sociais e queixas de problemas com os indicadores de práticas educativas, recursos do ambiente familiar e depressão materna.

\section{Método}

Adotou-se um delineamento caso-controle, transversal, correlacional.

\section{Aspectos Éticos}

Este projeto foi aprovado pelo Comitê de Ética em Pesquisa da universidade em que foi desenvolvido. É parte de um projeto maior com o título Saúde, Habilidades Sociais Conjugais e Educativas Parentais: comparações quanto a escolaridade, gênero e problemas de comportamento (Processo no. 5826/46/01/10).

\section{Participantes}

Participaram deste estudo mães de 32 crianças com problemas internalizantes e de 32 crianças sem problemas, totalizando 64 crianças avaliadas. As crianças sem problemas de internalização não apresentavam qualquer outro problema de comportamento e foram pareadas por sexo e por escolaridade, tal como descrito adiante em percurso amostral. Em cada grupo havia 15 meninos e 17 meninas e 17 crianças do Ensino Infantil e 15 do Ensino Fundamental. A idade média das crianças foi de 6 anos ( $\mathrm{DP}=2,8)$. A idade média das respondentes foi de 33 anos $(\mathrm{DP}=7,74)$. Comparações de idades de crianças e respondentes não diferenciaram os grupos (Teste $t$ ). A idade na pré-escola variou de 4 a 6 anos (média $=3,97 ; \mathrm{DP}=1,17$ ). Na idade escolar a variação foi de 7 a 11 anos (média $=8,19$; $\mathrm{DP}=2,69$ ).

As características demográficas dos familiares não diferenciaram estatisticamente os grupos com e sem problemas internalizantes (Teste do Qui-Quadrado). Quanto ao estado civil $82 \%$ das famílias apresentam união estável, $10 \%$ das mães são solteiras, $8 \%$ divorciadas. Quanto à escolaridade, $32 \%$ da amostra possui o primeiro grau (completo ou incompleto), $38 \%$ têm o segundo grau (completo ou incompleto) e 29\%, o terceiro grau (completo ou incompleto). Cinquenta por cento das mães trabalha fora. Quanto à renda familiar, em salários mínimos, os dados são: $10 \%$ - até um salário; $25 \%$ - 2 salários mínimos; $28 \%-3$ salários mínimos; $13 \%$ - 4 salários; $13 \%$ - 5 salários; $8,9 \%$ acima de 6 salários.

\section{Percurso amostral}

Foi solicitado a 34 professoras de Escolas Municipais de Ensino Infantil - EMEI e a 32 de Escolas Municipais de Ensino Fundamental - EMEF que indicassem duas crianças, da sala sob sua responsabilidade, uma que consideravam ter problemas de comportamento e outra sem problemas de comportamento. Dessas indicações resultou uma amostra de conveniência com 172 crianças pré-escolares e escolares, sendo identificadas 87 crianças com problemas de comportamento, segundo a avaliação das mães no CBCL-Child Behavior Checklist (descrito na seção Instrumentos). Nesta subamostra foram identificadas 32 crianças que apresentavam apenas problemas de comportamento internalizante, número que corresponde a $39 \%$ da subamostra com problemas e $19,76 \%$ da amostra total. Da subamostra de 85 crianças sem problemas de comportamento foram selecionadas 32 crianças pareadas por sexo e escolaridade. Para ser considerada sem problemas, a criança não poderia receber classificação clínica em qualquer das grandes escalas do CBCL (internalizante, externalizante e total).

\section{Instrumentos}

1. Roteiro de Entrevista de Habilidades Sociais Educativas Parentais (RE-HSE-P, Bolsoni-Silva, Loureiro \& Marturano, 2014), é uma entrevista semiestruturada que avalia práticas educativas relacionadas à comunicação, ao estabelecimento de limites e à expressão de sentimentos, classificando-as em positivas (por exemplo, conversar sobre assuntos de interesse da criança, expressar afeto, solicitar mudança de comportamento) ou negativas (por exemplo, bater, xingar, gritar para estabelecer limites). O RE-HSE-P também avalia queixas de comportamentos 
problemas (por exemplo, desobediência), habilidades sociais infantis e a diversidade de contextos em que as interações entre pais e crianças ocorrem (por exemplo, se a conversação da mãe com a criança ocorre em diversos momentos do dia). Precedendo as questões específicas são coletadas informações sobre: escolaridade, estado civil, nível socioeconômico e trabalho externo. O alfa para a amostra deste estudo foi de 0,846 . O instrumento diferencia crianças com e sem problemas de comportamento (Bolsoni-Silva et al., 2014).

2. CBCL "Child Behavior Checklist" (Inventário de Comportamentos da Infância e Adolescência, Achenbach \& Rescorla, 2001) para pré-escolares e escolares (4 a 18 anos) que investiga, a partir do relato de pais, a frequência de 113 respostas indicativas de problemas de comportamento. Os resultados são organizados em problemas internalizantes, externalizantes e totais. Bardin, Mari e Caeiro (1995) encontraram satisfatórios critérios de teste-positividade e de morbidade para os perfis clínico e não clínico.

3. Questionário de Respostas Socialmente Habilidosas para Pais (QRSH-Pais), que avalia a frequência de respostas socialmente habilidosas, segundo relatos de pais e mães (Bolsoni-Silva, Marturano \& Loureiro, 2011). O instrumento, com alfa de 0,82 , discriminou crianças com e sem problemas de comportamento segundoa avaliação de mães e de professores.

4. Inventário dos Recursos do Ambiente Familiar - RAF (Marturano, 2006). Avalia a qualidade do ambiente familiar em dez tópicos que compõem três categorias: apoio nas rotinas escolares (por exemplo, supervisão da lição de casa), oportunidades de interação (por exemplo, passeios) e recursos no ambiente físico (por exemplo, brinquedos). O escore total corresponde à soma dos escores obtidos nos dez tópicos. A autora refere consistência interna satisfatória e associação positiva com indicadores de competência social e ajustamento.

5. PHQ-9 - Questionário Sobre a Saúde do Paciente-9 (Patient Health Questionnaire). O PHQ-9 é um módulo baseado diretamente nos critérios diagnósticos para Desordem de Depressão Maior do DSM-IV proposto e validado por Spitzer et al. (1999) e por Kroenke, Spitzer e Williams (2001) que possibilita tanto o rastreamento de sinais e sintomas da Depressão Maior atual como a classificação de níveis de gravidade, leve, moderada e grave, sendo que quanto maior o escore mais indicadores de problemas. Estudo de fidedignidade foi realizado por Osório, Mendes, Crippa e Loureiro (2009), com indicadores satisfatórios.

\section{Procedimentos de coleta de dados}

Foi obtida a aprovação prévia da Secretaria de Educação Infantil de cidade do interior paulista; após, Escolas de Educação Infantil (EMEI) e de Ensino Fundamental (EMEF) foram contatadas sendo apresentados os objetivos do presente trabalho para a diretora ou a coordenadora pedagógica e também para professoras, que ao aceitarem participar assinaram um termo de Consentimento Livre e Esclarecido. Foi solicitado a 34 professoras de EMEI e a 32 de EMEF que indicassem duas crianças, da sala sob sua responsabilidade, uma que consideravam ter problemas de comportamento e outra sem problemas de comportamento.

Com base nessas informações, as famílias de crianças indicadas pelas professoras foram convidadas a participar da pesquisa. As mães que aceitaram participar assinaram um Termo de Consentimento Livre e Esclarecido. As entrevistas foram conduzidas em locais de melhor acesso a elas (na própria casa, na escola ou no Centro de Psicologia Aplicada da universidade), momento em que responderam aos instrumentos em duas sessões de aplicação.

\section{Procedimento de tratamento e análise de dados}

Com base nos cinco instrumentos aplicados, foram derivadas medidas de problemas de comportamento (CBCL,Queixas do RE-HSE-P), habilidades sociais (QRSH, RE-HSE-P), práticas positivas (RE-HSE-P), práticas negativas (RE-HSE-P), qualidade do ambiente (RAF, Diversidade de Contextos do RE-HSE-P) e depressão materna (PHQ-9).

A sequência de tratamento dos dados foi: (a) lançar os dados do CBCL no Software ASEBA para codificar os comportamentos das crianças; (b) organizar os participantes em grupos de acordo com ter ou não problemas de comportamento internalizante; (c) descrever e comparar os problemas de comportamento internalizante considerando a indicação das professoras, o sexo e a escolaridade (Teste do Qui-quadrado); (d) comparar os grupos com e sem problemas de comportamento internalizante quanto às variáveis do estudo (Teste $t$ de Student); (e) comparar subgrupos de sexo e escolaridade em relação às variáveis do estudo, separadamente no grupo com problemas internalizantes e no grupo sem problemas (Teste do Qui-Quadrado); (f) correlacionar as variáveis do estudo para o grupo com problemas internalizantes (Teste de Correlação de Pearson). Foi considerada significância a 5\%. 


\section{Resultados}

A seção de resultados apresenta a prevalência dos problemas internalizantes, considerando a indicação das professoras, o sexo e a escolaridade. Na sequência são apresentados os achados das comparações de grupo e das correlações conduzidas.

De acordo com a Tabela 1 não há associação estatisticamente significativa entre a indicação oral da professora quanto à criança ter ou não problema de comportamento e a classificação diagnóstica do CBCL em internalização. Nota-se que a probabilidade associada ao valor de $\chi^{2}$ se aproxima de 0,05 , porém não sinaliza concordância, mas antes discordância entre a indicação da professora e a classificação no CBCL, visto que 19 crianças, em torno de $60 \%$ da amostra, foram consideradas pela professora como não tendo problemas de comportamento e apresentaram, do ponto de vista dos pais, problemas de internalização. A Tabela 2 apresenta a frequência de problemas internalizantes nas síndromes do CBCL, de acordo com sexo e escolaridade.

Conforme a Tabela 2 os sintomas mais frequentes são de ansiedade/depressão (66,66\%) e ansiedade (63,63\%). Problemas somáticos aparecem em 42,42\% da amostra e afetivos em $30,30 \%$ das crianças. O teste do Qui-Quadrado não detectou diferenças entre meninos e meninas. Nas comparações por escolaridade, apenas a subescala ansiedade foi estatisticamente mais frequente entre os escolares. A Tabela 3 descreve os resultados da comparação de variáveis da criança e da família nos grupos com e sem problemas internalizantes.

De acordo com a Tabela 3 as práticas negativas e as queixas de problemas de comportamento identificadas com o RE-HSE-P tiveram maiores médias para o grupo com problemas internalizantes. Em contrapartida, as habilidades sociais infantis avaliadas pelo QRSH foram mais presentes no grupo sem problemas.

Análises adicionais (Teste U Mann-Whitney) foram conduzidas considerando separadamente os grupos com e sem problemas de comportamento internalizantes, para verificar associações com sexo e escolaridade. São descritos os resultados que diferenciaram os grupos. No grupo com problemas internalizantes, obteve-se diferença de sexo para práticas negativas, com maiores médias para meninos (média/DP menino $=7,80 / 4,23$; média/DP menina $=4,88 / 2,59, p=0,040)$. No grupo sem problemas nenhuma variável diferenciou os grupos, seja nas comparações por sexo ou por escolaridade.

TABELA 1

Comparação entre a Indicação Oral da Professora e a Avaliação Diagnóstica do CBCL para Internalização (Teste Qui-Quadrado)

\begin{tabular}{lcccc}
\hline & & \multicolumn{2}{c}{ Indicação da professora } & \multirow{2}{*}{ Total } \\
\cline { 3 - 4 } & Sem PCI & $\begin{array}{c}\text { Com problema } \\
\text { de comportamento }\end{array}$ & $\begin{array}{c}\text { Sem problema } \\
\text { de comportamento }\end{array}$ & \\
\hline \multirow{2}{*}{ CBCL } & Com PCI & $13(15,63 \%)$ & $27(84,38 \%)$ & $32(100 \%)$ \\
& Pearson Chi-Square & & $19(59,38 \%)$ & $32(100 \%)$ \\
\hline
\end{tabular}

PCI: Problemas internalizantes em nível clínico no CBCL.

TABELA 2

Frequência de Problemas Internalizantes $(\mathrm{N}=33)$ nas Síndromes do CBCL, de Acordo com Sexo e Escolaridade (Teste Qui-Quadrado)

\begin{tabular}{lcccc}
\hline \multicolumn{1}{c}{ Problemas CBCL } & $\begin{array}{c}\text { Meninos } \\
(N=16)\end{array}$ & $\begin{array}{c}\text { Meninas } \\
(N=17)\end{array}$ & $\begin{array}{c}\text { Total } \\
(N=33)\end{array}$ & $p$ \\
\hline Ansiedade/depressão & $10(62,50 \%)$ & $13(76,47 \%)$ & $23(69,70 \%)$ & 0,683 \\
Ansiedade & $12(75,00 \%)$ & $12(70,59 \%)$ & $22(66,67 \%)$ & 0,698 \\
Somáticos & $6(37,50 \%)$ & $8(47,06 \%)$ & $14(42,42 \%)$ & 0,733 \\
Afetivos & $7(43,75 \%)$ & $3(17,65 \%)$ & $10(30,30 \%)$ & 0,111 \\
& $E M E I$ & $E M E F$ & Total & $p$ \\
Ansiedade/depressão & $(N=17)$ & $(N=16)$ & $(N=33)$ & 0,683 \\
Ansiedade & $13(76,47 \%)$ & $10(62,50 \%)$ & $23(69,70 \%)$ & 0,052 \\
Somáticos & $9(52,94 \%)$ & $15(93,75 \%)$ & $24(72,73 \%)$ & 0,120 \\
Afetivos & $10(58,82 \%)$ & $4(25,00 \%)$ & $14(42,42 \%)$ & 0,111 \\
\hline
\end{tabular}


TABELA 3

Variáveis da Criança e da Família: Médias (Desvios-Padrão) nos Grupos com e sem Problemas Internalizantes. (Teste $t$ de Student).

\begin{tabular}{lcccc}
\hline \multicolumn{1}{c}{ Variáveis } & Grupo com PCI & Grupos em PCI & $T$ & $p$ \\
\hline Habilidades sociais - RE-HSE-P & $19,00(6,88)$ & $19,56(6,19)$ & $-0,344$ & 0,732 \\
Habilidades sociais - QRSH & $28,96(7,18)$ & $46,78(49,48)$ & $-2,015$ & $0,048^{*}$ \\
Queixas comportamento - RE-HSE & $7,34(3,98)$ & $5,00(3,88)$ & 2,386 & $0,020^{*}$ \\
Práticas positivas & $20,22(6,59)$ & $20,78(5,80)$ & $-0,362$ & 0,718 \\
Práticas negativas & $12,68(6,39)$ & $8,78(4,62)$ & 2,802 & $0,007^{* *}$ \\
Qualidade do ambiente & & & \\
$\quad$ Contextos - RE-HSE & $13,31(6,11)$ & $12,16(6,61)$ & 0,726 & 0,470 \\
$\quad$ Recursos - RAF & $73,97(13,99)$ & $71,59(25,55)$ & 0,461 & 0,647 \\
$\quad$ Depressão materna & $4,47(3,45)$ & $2,84(3,71)$ & 1,814 & 0,075 \\
\hline
\end{tabular}

PCI: Problemas internalizantes em nível clínico no CBCL; RE-HSE: Roteiro de Entrevista de Habilidades Sociais Educativas Parentais; QRSH: Questionário de Respostas Socialmente Habilidosas para Pais; RAF: Inventário de Recursos do Ambiente Familiar. $* p<0,05 ; * * p<0,01$.

TABELA 4

Correlações, no Grupo com Problemas de Comportamento Internalizantes, entre Práticas Educativas, Habilidades Sociais Infantis, Queixas de Problemas de Comportamento, Recursos do Ambiente, Relacionamento Conjugal e Depressão Materna (Teste de Correlação de Pearson).

\begin{tabular}{|c|c|c|c|c|c|c|c|c|}
\hline & 1 & 2 & 3 & 4 & 5 & 6 & 7 & 8 \\
\hline 1. Habilidades sociais - RE-HSE & 1 & $-0,023$ & 0,045 & $0,687 * *$ & $0,363^{*}$ & $0,583 * *$ & 0,221 & 0,138 \\
\hline 2. Habilidades sociais $-\mathrm{QRSH}$ & & 1 & $-0,339$ & 0,139 & $-0,049$ & 0,230 & 0,106 & $-0,350 *$ \\
\hline 3. Problemas de comportamento relatados & & & 1 & $-0,058$ & $0,413^{*}$ & 0,281 & 0,155 & 0,192 \\
\hline 4. Práticas positivas & & & & 1 & 0,149 & $0,581 * *$ & $0,355^{*}$ & 0,079 \\
\hline 5. Práticas negativas & & & & & 1 & 0,325 & $-0,094$ & 0,187 \\
\hline 6. Qualidade ambiente - Contextos RE-HSE & & & & & & 1 & $0,407 *$ & 0,026 \\
\hline 7. Qualidade ambiente - Recursos RAF & & & & & & & 1 & $-0,164$ \\
\hline 8. Depressão materna & & & & & & & & 1 \\
\hline
\end{tabular}

RE-HSE: Roteiro de Entrevista de Habilidades Sociais Educativas Parentais; QRSH: Questionário de Respostas Socialmente Habilidosas para Pais; RAF: Inventário de Recursos do Ambiente Familiar.

* A correlação é significativa no nível 0,05 (2 extremidades); **A correlação é significativa no nível 0,01 (2 extremidades).

A Tabela 4 apresenta as correlações entre práticas educativas, habilidades sociais infantis, queixas de problemas de comportamento, recursos do ambiente e depressão materna. Essas correlações foram obtidas no grupo com problemas de comportamento internalizante.

A maioria das correlações encontradas no grupo com problemas internalizantes foram diretas. Três variáveis do ambiente apresentaram-se assim interrelacionadas: práticas educativas positivas, diversidade de contextos de interação e recursos do ambiente familiar. Dentre elas, práticas educativas positivas e diversidade de contextos de interação foram diretamente correlacionadas com habilidades sociais infantis relatadas na entrevista. Entretanto, habilidades sociais relatadas também tiveram correlação direta, ainda que fraca, com práticas educativas negativas. Queixas de problemas de comportamento trazidas na entrevista foram moderadamente correlacionadas com práticas negativas. Habilidades sociais do QRSH foram moderadamente e inversamente correlacionadas com depressão materna.

\section{Discussão}

Esta pesquisa teve por objetivos comparar e correlacionar indicadores de práticas educativas, recursos do ambiente familiar, comportamento infantil e depressão materna de crianças com e sem problemas internalizantes, diferenciadas por sexo e escolaridade. Os principais resultados foram: (a) práticas negativas foram mais frequentes no grupo com problemas de comportamento internalizante e, ao contrário, as habilidades sociais das crianças, mais frequentes no grupo sem problemas; (b) práticas negativas correlacionaram-se a queixas de problemas e práticas positivas foram correlacionadas com habilidades sociais da criança e recursos do ambiente; (c) quanto maior o escore de depressão materna, menos frequentes 
as habilidades sociais infantis; (d) crianças escolares tendem a apresentar mais problemas de ansiedade que crianças pré-escolares.

Verifica-se, neste estudo, que mais de $50 \%$ das crianças com problemas internalizantes não apresentavam problemas do ponto de vista das professoras. Esse dado remete às considerações de Flett e Hewitt (2013) quanto à baixa identificação do problema pelos adultos. Quanto ao fato de aparentemente a percepção das mães ter sido mais discriminativa que a das professoras, cabe lembrar que problemas internalizantes em crianças e adolescentes são susceptíveis de causar significativo estresse para suas mães no curso do tempo (Barbot et al., 2014), o que poderia explicar a divergência.

$\mathrm{Na}$ avaliação das mães do grupo com problemas internalizantes, observou-se, como tendência, que a subescala ansiedade foi mais frequente nos escolares em comparação aos pré-escolares, o que condiz com resultados de estudos longitudinais mostrando trajetórias ascendentes de problemas internalizantes como típicas do desenvolvimento nessa faixa etária (Fanti \& Henrich, 2010; Ghandour et al., 2010).

Em contrapartida, no grupo com problemas internalizantes não foram observadas diferenças de sexo nas síndromes do CBCL; esse resultado aparentemente está em desacordo com a literatura, que associa problemas internalizantes ao sexo feminino (APA, 2000; Fanti \& Henrich, 2010). No entanto, é preciso considerar que a maior presença de internalização no sexo feminino tem sido encontrada em estudos epidemiológicos. Em grupos clínicos ou identificados com algum transtorno não se observou o viés feminino (Munkvold \& Lundervold, 2011; Graziano et al., 2011), tal como no presente estudo, em que as comparações de sexo foram feitas entre crianças com manifestações internalizantes em nível clínico no CBCL. Parece ainda haver interação entre sexo e idade. No estudo de Ghandour et al. (2010) sobre crianças com problemas de saúde física, emocional, comportamental ou de desenvolvimento, gênero só foi significativamente associado a sintomas internalizantes a partir dos 12 anos, não se observando tal associação em crianças de 3 a 11 anos, faixa etária próxima à da amostra do presente estudo.

No exame das condições de risco e proteção para problemas internalizantes, a pesquisa considerou variáveis da criança e da família, segundo o relato das mães. No que se refere às variáveis da criança, as queixas de problemas de comportamento, identificadas com o RE-HSE-P, tiveram maiores médias para o grupo com problemas internalizantes em relação ao grupo sem problemas. Esses resultados são condizentes com os de Burt e Roisman (2010), que identificaram, nos problemas de comportamento precoces, precursores de problemas internalizantes. Já as habilidades sociais infantis avaliadas pelo QRSH foram mais presentes no grupo sem problemas, corroborando a interpretação de que habilidades sociais mais desenvolvidas podem proteger as crianças contra transtornos de ansiedade, tal como constataram Wichstrom et al. (2013) ao seguirem crianças dos 4 até os 6 anos.

Os resultados sugestivos de que crianças com problemas internalizantes apresentam déficits de habilidades sociais são coerentes com os de estudos longitudinais que mostram trajetórias de internalização associadas a habilidades sociais mais pobres (Dozois \& Westra, 2004; Burt \& Roisman, 2010; Fanti \& Henrich, 2010), endossando a noção de que a internalização de sintomas é influenciada por interações entre os indivíduos e seu contexto ambiental (Letcher et al., 2012).

Dentre as variáveis do ambiente incluídas no estudo, o fator de risco configurado pela depressão materna não foi associado a problemas internalizantes nas crianças, ao contrário do que se encontra bem estabelecido na literatura (Pizeta et al., 2013). Em contrapartida, habilidades sociais do QRSH foram moderadamente e inversamente correlacionadas com depressão materna no grupo com problemas internalizantes. Esse resultado corrobora o achado de Boyd e Waanders (2013), de que habilidades sociais têm efeito protetor contra problemas internalizantes em presença do risco representado pela depressão materna.

As práticas educativas maternas, quando negativas, foram mais presentes no grupo com problemas internalizantes, com maiores médias para os meninos deste grupo. Os resultados de comparação de grupos confirmam em parte a literatura (Trepat et al., 2014; Xing \& Wang, 2013), uma vez que as práticas negativas (com maior frequência para meninos), tanto quanto os comportamentos problema relatados, sobretudo agressividade e desobediência, diferenciaram os grupos. Diversos autores afirmam que o uso de práticas negativas, como aversividade, excesso de superproteção e críticas aumentam a chance de a criança apresentar tais problemas (APA, 2000; Dozois \& Westra, 2004; Essau, 2004; Hudson et al., 2004). A correlação moderada entre práticas educativas negativas e queixas de problemas de comportamento no grupo com problemas internalizantes, observada no presente estudo, corrobora essa visão e confirma resultados de estudos prévios (Bolsoni-Silva et al., 2011; Hudson et al., 2004; Trepat et al., 2014; Xing \& Wang, 2013).

As práticas educativas positivas não diferenciaram os grupos, no entanto, figuraram em diversas correlações 
no grupo com problemas internalizantes. Elas foram correlacionadas com diversidade de contextos de interação (Bolsoni-Silva et al., 2014) e recursos do ambiente familiar (Marturano, 2006). Também foram diretamente correlacionadas com habilidades sociais infantis relatadas na entrevista. Analogamente, a qualidade do ambiente familiar, assinalada pela diversidade de contextos de interação, foi diretamente correlacionada com habilidades sociais infantis relatadas na entrevista. Essa configuração de resultados é compatível com uma interpretação do efeito protetor das práticas educativas positivas (Boyd \& Waanders, 2013; Luyckx et al., 2011) e da qualidade do ambiente familiar (Kelley et al., 2011; Rijlaarsdam et al., 2013), pela via do fortalecimento das habilidades sociais da criança (Bolsoni-Silva et al., 2011). A afirmativa de que as práticas positivas previnem o surgimento de problemas de comportamento e promovem habilidades sociais infantis (Bolsoni-Silva et al., 2011) foi em parte confirmada, pois tais relações apareceram nas análises de correlações, ainda que não tenham sido detectadas nas comparações de grupos.

As habilidades sociais relatadas na entrevista tiveram correlação direta, ainda que fraca, com práticas educativas negativas, o que discorda do esperado pela literatura (Bolsoni-Silva et al., 2014). No entanto, destaca-se que mesmo crianças com problemas de comportamento possuem repertório de habilidades sociais (Bolsoni-Silva et al., 2011) e que, em grupos clínicos para internalização, verifica-se que as mães podem deixar de estimular ou até mesmo punir a emissão de comportamentos de habilidades sociais, como constatado por Orti (2014).

\section{Considerações Finais}

A pesquisa identificou problemas internalizantes, especialmente ansiedade e comorbidade ansiedade/ depressão. As práticas negativas e as queixas de problemas foram mais frequentes no grupo com problemas e, ao contrário, as habilidades sociais das crianças, foram mais frequentes no grupo sem problemas. As práticas negativas correlacionaram-se a queixas de problemas e as práticas positivas foram correlacionadas às habilidades sociais da criança e recursos do ambiente. Quanto maior o escore de depressão materna, menos frequentes são as habilidades sociais infantis. Meninas e meninos apresentam igualmente internalização e as pré-escolares têm menos problemas de ansiedade que as escolares.

A investigação tem por ponto forte o estudo de internalização, sem comorbidades com outros problemas, a partir de um instrumento diagnóstico. Outros aspectos a serem destacados são: a composição de um grupo sem problemas pareado por sexo e escolaridade, controlando tais variáveis; a mensuração de múltiplas variáveis preditivas de problemas de internalização que puderam ser comparadas e correlacionadas. Conclui-se que o estudo traz informações pertinentes sobre a avaliação simultânea de diferentes fatores de risco e de proteção no estudo da internalização, bem como o controle de variáveis, garantido no método. Tais achados sinalizam caminhos para a elaboração e aplicação de procedimentos de prevenção. A prevenção é relevante porque problemas internalizantes podem ser agravados, no curso do desenvolvimento, implicando em problemas de saúde mental na adolescência e vida adulta. Os resultados desta investigação sugerem, como alvos prioritários de intervenção para prevenir ou atenuar problemas internalizantes na meninice, as práticas educativas maternas e as habilidades sociais infantis, por meio de ações capazes de obstar o desenvolvimento de transtornos ansiosos e/ou depressivos ou de comorbidade com outros transtornos. Reitera-se a importância de estudar múltiplas variáveis simultaneamente de forma a compreender melhor o fenômeno problema internalizante infantil, o que se pretendeu com a presente pesquisa.

Considera-se como limitações, o número reduzido de participantes, a coleta em apenas um município e a falta de mensuração por observação direta. Tais aspectos podem ser garantidos em futuras pesquisas.

\section{Referências}

Achenbach, T. M. \& Rescorla, L. A. (2001). Manual for the ASEBA School-Age Forms \& Profiles. Burlington, VT: University of Vermont, Research Center for Children, Youth, \& Families.

Achenbach, T. M., Becker, A., Döpfner, M., Heiervang, E., Roessner, V., Steinhausen, H. C. et al. (2008). Multicultural assessment of child and adolescent psychopathology with ASEBA and SDQ instruments: research findings, applications, and future directions. Journal of Child Psychology and Psychiatry, 49(3), 251-275. http://dx.doi.org/10.1111/j.14697610.2007.01867.x

American Psychiatric Association (2000). Diagnostic and statistics manual of mental disorders (4⿳亠口了 ed.). Washington, DC: APA. 
Barbot, B., Crossman, E., Hunter, S. R., Grigorenko, E. L., \& Luthar, S. S. (2014). Reciprocal Influences Between Maternal Parenting and Child Adjustment in a High-Risk Population: A 5-Year Cross-Lagged Analysis of Bidirectional Effects. American Journal of Orthopsychiatry, 84(5), 567-580. http://dx.doi.org/10.1037/ort0000012

Bordin, I. A. S., Mari, J. J., \& Caeiro, M. F. (2003).Validação da versão brasileira do "Child Behavior Checklist" (CBCL) (Inventário de Comportamentos da Infância e Adolescência): dados preliminares. Revista ABP-APAL, 17(2), 55-66.

Bolsoni-Silva, A. T., Loureiro, S., \& Marturano, E. M. (2014). Roteiro de entrevista de habilidades sociais educativas parentais (RE-HSE-P). Manual Técnico. São Carlos, SP: Suprema.

Bolsoni-Silva, A. T., Marturano, E. M., \& Loureiro, S. R. (2011). Estudos de confiabilidade e validade do questionário de respostas socialmente habilidosas versão para pais - QRSH-Pais. Psicologia: Reflexão e Crítica, 24(2), 1-9.

Boyd, R. C. \& Waanders, C. (2013). Protective Factors for Depression Among African American Children of Predominantly Low-Income Mothers with Depression. Journal of Child and Family Study, 22(1), 85-95. http:// dx.doi.org/10.1007/s10826-012-9588-y

Burt, K. B. \& Roisman, G. I. (2010). Competence and psychopathology: cascade effects in the NICHD Study of Early Child Care and Youth Development. Development and Psychopathology, 22, 557-567. http://dx.doi.org/10.1017/ S0954579410000271

Dozois, J. A. \& Dobson, K. S. (2004). The prevention of anxiety and depression. Theory, research and practice. Washington, DC: American Psychological Association. http://dx.doi.org/10.1037/10722-000

Dozois, J. A. \& Westra, H. A. (2004). The nature of anxiety and depression: implications for prevention. In D. J. A. Dozois \& K. S. Dobson (Eds.). The prevention of anxiety and depression. Theory, research and practice (pp. 9-42). Washington, DC: American Psychology Association. http://dx.doi.org/10.1037/10722-002

Essau, C. A. (2004). Primary prevention of depression. In D. J. A. Dozois \& K. S. Dobson (Eds.). The The prevention of anxiety and depression. Theory, research and practice (pp. 185-204). Washington, DC: American PsychologyAssociation. http://dx.doi.org/10.1037/10722-008

Fanti, K. A. \& Henrich, C. C. (2010). Trajectories of pure and co-occurring internalizing and externalizing problems from age 2 to age 12: Findings from the National Institute of Child Health and Human Development Study of Early Child Care. Developmental Psychology, 46(5), 1159-1175. http://dx.doi.org/10.1037/a0020659

Flett, G. L. \& Hewitt, P. L. (2013). Disguised distress in children and adolescents "flying under the radar": Why psychological problems are underestimated and how schools must respond. Canadian Journal of School Psychology, 28, p. 12-27. http://dx.doi.org/10.1177/0829573512468845

Ghandour, R. M., Kogan, M. D., Blumberg, S. J., \& Perry,D. F. (2010). Prevalence and correlates of internalizing mental health symptoms among CSHCN. Pediatrics, 125(2), 269-277. http://dx.doi.org/10.1542/peds.2009-0622

Graziano, P., Geffken, G., \& McNamara. (2011). Atypical behaviors and comorbid externalizing symptoms equally predict social functioning in children with Attention-Deficit/Hyperactivity Disorder. Child Psychiatry and Human Development, 42, 377-389. http://dx.doi.org/10.1007/s10578-011-0224-7

Hudson, J. L., Flannery-Schroeder, E., \& Kendall, P. C. (2004).Primary prevention of anxiety disorders. In D. J. A. Dozois \& K .S. Dobson (Eds.). The Prevention of Anxiety and Depression: Theory, Research and Practic (101-130). Washington, DC: American PsychologyAssociation. http://dx.doi.org/10.1037/10722-005

Kelley, S. J., Whitley, D. M., \& Campos, P. E. (2011). Behavior problems in children raised by grandmothers: The role of caregiver distress, family resources, and the home environment. Children and Youth Services Review, 33, 2138-2145. http://dx.doi.org/10.1016/j.childyouth.2011.06.021

Kroenke, K., Spitzer, R. L., \& Williams, J. B.W. (2001). The PHQ-9. Validity of a Brief Depression Severity Measure. Journal of General Internal Medicine, 16(9), 606-613. http://dx.doi.org/10.1046/j.1525-1497.2001.016009606.x

Letcher, P., Sanson, A., Smart, D., \& Toumbourou, J. W. (2012). Precursors and Correlates of Anxiety Trajectories From Late Childhood to Late Adolescence. Journal of Clinical Child \& Adolescent Psychology, 41(4), 417-432. http:// dx.doi.org/10.1080/15374416.2012.680189

Luyckx, K., Tildesley, E. A., Soenens, B., Andrews, J. A., Hampson, S.E., Peterson, M., \& Duriez, B. (2011). Parenting and trajectories of children's maladaptive behaviors: A 12-year prospective community study. Journal of Clinical Child and Adolescent Psychology, 40(3), 468-478. http://dx.doi.org/10.1080/15374416.2011.563470

Marturano, E. M. (2006). O Inventário de Recursos do Ambiente Familiar. Psicologia: Reflexão e Crítica, 19(3), 498-506. http://dx.doi.org/10.1590/S0102-79722006000300019

Munkvold, L. H., Lundervold, A. J., \& Manger, T. (2011). Oppositional defiant disorder-gender differences in co-occurring symptoms of mental health problems in a general population of children. Journal of Abnormal ChildPsychology, 39, 577-587. http://dx.doi.org/10.1007/s10802-011-9486-6

Orti, N. P. (2014). Avaliação dos efeitos e variáveis do processo de intervenção com mães de crianças com problemas internalizantes. Dissertação de Mestrado não-publicada, Curso de Pós-Graduação em Psicologia do Desenvolvimento e Aprendizagem, Universidade Estadual Paulista, Bauru, SP.

Osório, F. L., Mendes, A. V., Crippa, J. A., \& Loureiro, S. R. (2009). Study of the discriminative validity of the phq-9 and phq-2 in a sample of brazilian women in the context of primary health care. Perspectives in Psychiatric Care, 45(3), 216-227. http://dx.doi.org/10.1111/j.1744-6163.2009.00224.x 
Pizeta, F. A., Silva, T. B. F., Cartafina, M. I. B., \& Loureiro, S. R. (2013). Depressão materna e riscos para o comportamento e a saúde mental das crianças: uma revisão. Estudos de psicologia (Natal), 18(3), 429-437. ISSN 1413-294X. http://dx.doi.org/10.1590/S1413-294X2013000300003

Rijlaarsdam, R., Stevens, W. J. M., van der Ende, J., Hofman, A., Jaddoe, V. W. V., Mackenbach, J. P., Verhulst, F. C., \& Tiemeier, H. (2013). Economic disadvantage and young children's emotional and behavioral problems: Mechanisms of risk. Journal of Abnormal Child Psychology, 41, 125-137. http://dx.doi.org/10.1007/s10802-012-9655-2

Spitzer, R. L., Williams, J. B., Kroenke, K, Linzer, M, DeGruy, F. V, Hahn, S. R, Brody, D., \& Johnson, J. G. (1994). Utility of a new procedure for diagnosing mental disorders in primary care. The PRIME-MD 1000 study. JAMA, 272(22), 1749-1756. http://dx.doi.org/10.1001/jama.1994.03520220043029

Trepat, E., Granero, R, \& Ezpeleta, L. (2014). Parenting practices as mediating variables between parents' psychopathology and oppositional defiant disorder in preschoolers. Psicothema, 26(4), 497-504.

Wichstrom, L., Belsky, J., \& Berg-Nielsen, T. S. (2013). Preschool predictors of childhood anxiety disorders: a prospective community study. Journal of Child Psychology and Psychiatry, 54(12), 1327-1336. http://dx.doi.org/10.1111/ jepp. 12116

Xing, X. \& Wang, M. (2013). Sex differences in the reciprocal relationships between mild and severe corporal punishment and children's internalizing problem behavior in a Chinese sample. Journal of Applied Developmental Psychology, 34, 9-16. http://dx.doi.org/10.1016/j.appdev.2012.09.004

\section{Autores:}

Alessandra Turini Bolsoni-Silva - Doutora, Universidade Estadual Paulista.

Sonia Regina Loureiro - Doutora, Faculdade de Medicina de Ribeirão Preto.

Edna Maria Marturano - Doutora, Faculdade de Medicina de Ribeirão Preto.

Endereço para correspondência:

Alessandra Turini Bolsoni-Silva

Faculdade de Ciências - UNESP - Campus de Bauru

Av. Eng. Luiz Edmundo Carrijo Coube, 14-01 - Vargem Limpa

17033-360 Bauru, SP, Brasil

<bolsoni@fc.unesp.br>

Recebido em: 20.05.2015

Aceito em: 28.03.2016 